\title{
LOD Score
}

National Cancer Institute

\section{Source}

National Cancer Institute. LOD Score. NCI Thesaurus. Code C16800.

The statistical probability that two genetic loci are likely to be inherited together. 